\title{
Annealing and Spectral Characteristics of Femtosecond Laser Inscribed Long Period Gratings written into a Photonic Crystal Fibre
}

\author{
T. Allsop ${ }^{\text {a }}$ K. Kalli ${ }^{\#}$ K. Zhou, M. Dubov, Y. Lai, D.J. Webb, I. Bennion \\ ${ }^{a}$ Photonics Research Group, Aston University, Aston Triangle, Birmingham, B4 7ET, U.K. \\ "Photonics Research Laboratory, Cyprus University of Technology, Cyprus \\ Telephone: (+44)121-2043484 \\ Fax: $\quad(+44) 121-359-0156$ \\ email: t.d.p.allsop@aston.ac.uk
}

\begin{abstract}
A series of LPGs with the same period was inscribed by femtosecond laser into photonic crystal fibre with various powers. All suffered post-fabrication spectral evolution at low temperatures, apparently related to inscription power.
\end{abstract}

KEYWORDS: Photonic Crystal Fibre, Annealing, Femtosecond laser, long period grating

\section{INTRODUCTION}

Long period gratings are currently the subject of considerable research interest due to their potential applications as filters and as sensing devices, responsive to strain, temperature, bending and refractive index [1]. In contrast to the more mature fibre Bragg grating sensors, LPGs are intrinsically sensitive to bending and refractive index. Perhaps more importantly, the fibre design and choice of grating period can have a considerable influence over the sensitivity to the various parameters, for example allowing the creation of a bend sensor with minimal temperature cross-sensitivity. This control is not possible with FBG sensors[2]. Recently considerable interest has been shown in using photonic crystal fibre (PCF) in conjunction with grating structures as fibre sensing devices; this is due to the "endless" geometric and material variations possible [3-4]. Particular interest is being shown in writing LPGs into PCF. Whilst the sensitivity of LPGs is an advantage over fibre Bragg gratings, there can be problems of cross-sensitivity between parameters with the most common being temperature when combined with another parameter which is of interest [5]. It has been shown [6] that LPGs in PCF can have very low temperature sensitivity thus reducing cross-sensitivity/cross-talk resulting in a sensor with greater resolution. One of the major problems to date is the inscription of the LPG into the PCF due to the fact that the majority of PCFs are made from pure fused-silica, which is not photosensitive to UV and thus traditional UV inscription techniques are not applicable. Several other inscription approaches have been used such as the electricarc method [6], $\mathrm{CO}_{2}$ lasers [7] and femtosecond lasers [8] to overcome this problem with the femtosecond laser technique being recognised as possibly the most versatile.

With the increasing usage of femtosecond lasers to fabricate LPGs in PCF, we feel it important to study the temporal spectral stability of such fibre devices. We discovered that all the LPGs we produced exhibited post-fabrication spectral evolution at room temperature as well as at higher temperatures along with polarisation dependence, all of which seem to be related to the inscription power used.

\section{FABRICATION OF GRATING DEVICES}

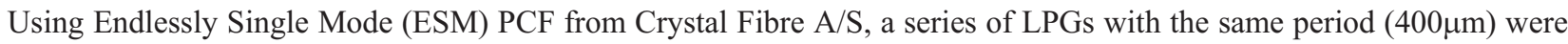
fabricated with inscription pulse energies ranging from 410 to $650 \mathrm{~nJ}$, requiring various lengths to obtain maximum strength attenuation bands. The refractive index changes to create the LPG are introduced by a NIR femtosecond laser (800nm Spitfire/Evolution titanium/sapphire system, Spectra-Physics Lasers). The femtosecond laser radiation was focused at a predetermined point near the core with $\mathrm{a} \times 100(\mathrm{NA}=0.55)$ microscope objective, while the fibre was moved in a direction parallel to the fibre axis. The translation speed was $100 \mu \mathrm{ms}^{-1}$. A cylindrical lens ( $\left.\mathrm{f}=100 \mathrm{~mm}\right)$ was put in the proximity of the microscope objective to shape the laser beam at the point of inscription. The femtosecond laser system produced a $1 \mathrm{kHz}$ train of $150 \mathrm{fs}$ pulses at $800 \mathrm{~nm}$, see Figure 1, other details of the experimental set-up can be found elsewhere ${ }^{8}$.

As a result of previous investigations into the spectral characteristics of femtosecond laser inscribed LPGs ${ }^{9}$ the light output from a broadband source was polarised and then a polarisation controller was used to change the polarisation of the light illuminating the LPGs (see figure 1b). The polarisation was constantly optimised to maximise coupling of the illuminating light into one of the attenuation bands present in the transmission spectrum of the LPG to observe the 
growth of the LPGs' attenuation bands during fabrication. Two examples of the transmission spectra are shown in figure $2 \mathrm{~b}$ and $2 \mathrm{c}$.

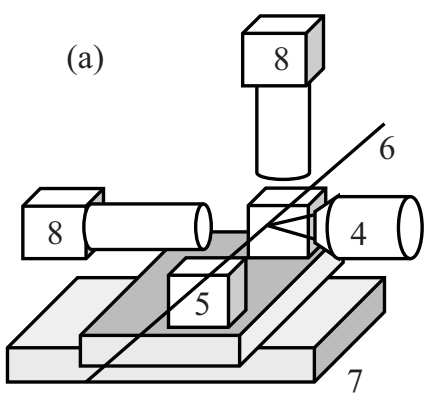

(b)
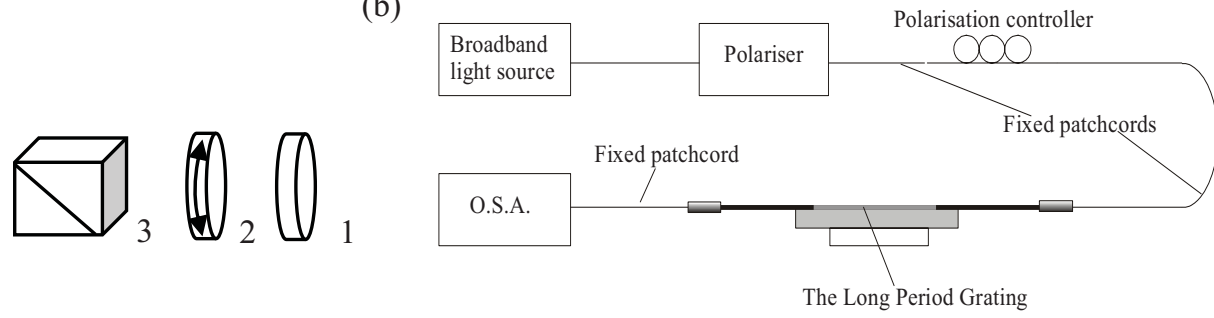

Figure 1 (a). Optical layout of inscription scheme. The light passes a shutter (1), a half-wave plate (2), Glan prism (3), x100 long working distance microscopic objective (4) before being focused in the fibre core (6). Two alignment 3D translation stages are mounted on top of the high precision computer controlled 2D-stage (7). We use two CCD-cameras (8) with optical zooming system for alignment and on-line monitoring of the inscription process. (b) A schematic of the apparatus used to investigate the transmission spectra of the LPGs during fabrication.
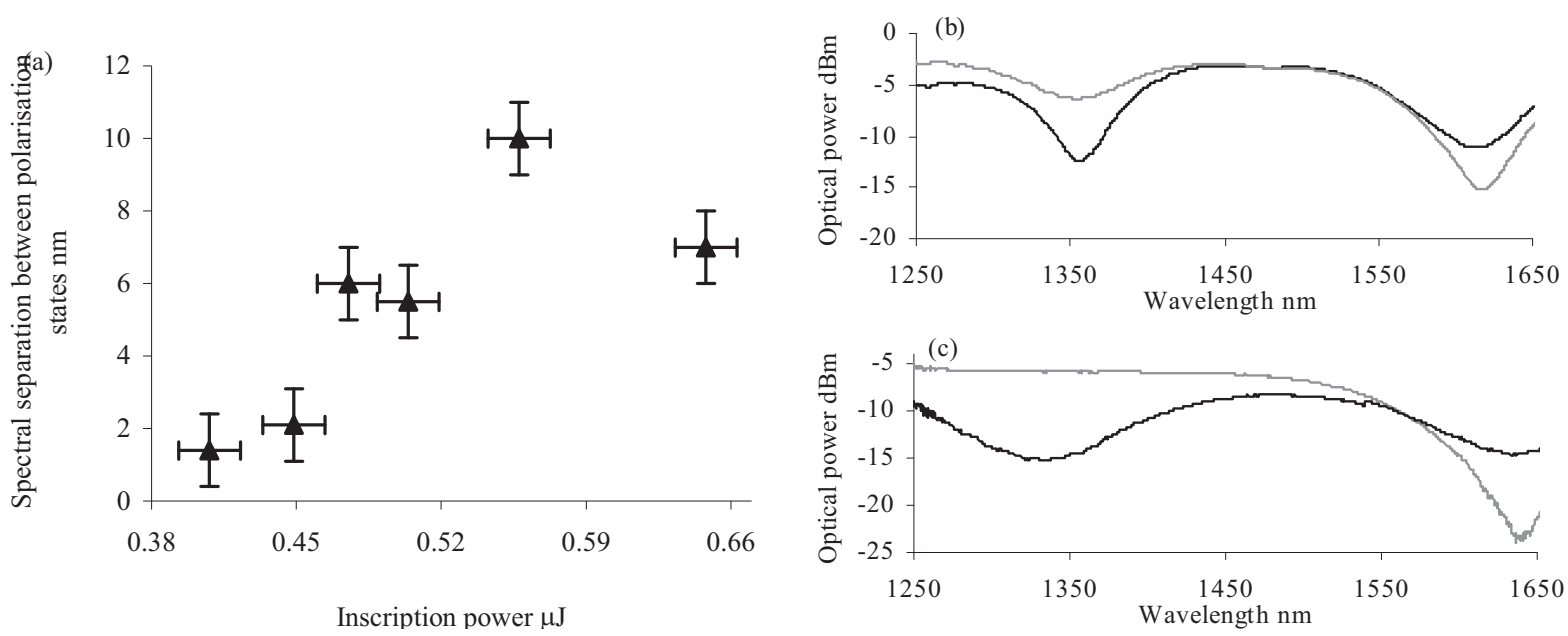

Figure 2. Examples of transmission spectra of two LPGs (period $400 \mu \mathrm{m}$ ) fabricated in PCF (ESM) showing the polarisation dependence, (a) The maximum observed wavelength separation of the attenuation band due to polarisation as a function of inscription energy , (b) power $500 \mathrm{~nJ}$, grating length of $7 \mathrm{~mm}$. (c) power $650 \mathrm{~nJ}$, grating length of $4 \mathrm{~mm}$

The polarisation dependence appears to have two main contributions one relating to the symmetry of the inscription (work to be published elsewhere) and the other to the power of inscription. The birefringence ranged from $10.2 \mathrm{~nm}$ to $1.4 \mathrm{~nm}$ over the power range used, see figure $2 \mathrm{a}$. This is discussed below.

\section{ANNEALING STUDIES}

\section{Annealing studies at room temperature}

The work so far on annealing has been carried out at room temperature and $80^{\circ} \mathrm{C}$; these temperatures being selected due to the fact that they are commonly used for the annealing of gratings in standard SMF. The experimental arrangement for measurements taken at room temperature is shown in figure $1 \mathrm{~b}$; at $80^{\circ} \mathrm{C}$ the LPGs were placed into an insulated box with a Peltier cooler. At room temperature it was observed that LPGs had post-fabrication spectral evolution. For all observed LPGs the general trends appear to be the same with an initial red wavelength shift followed by a further blue wavelength shift. The largest spectral shifts occurred in the first 120 hours of fabrication with the maximum observed wavelength shifts linked with the LPGs inscribed with the lowest powers. An example of the temporal response of the LPGs' transmission spectra is shown in figure $3 \mathrm{a}$ with the wavelength shift shown in figure $3 \mathrm{~b}$. For all LPGs observed at room temperature, the maximum wavelength shift observed is shown in figure $3 \mathrm{c}$ (note that the two results in the circle are 
measurements not taken at the same time as the other LPGs; one being at only 90 hours and the other at 147 hours after inscription). The other LPGs were measured at approximately 120 hours.
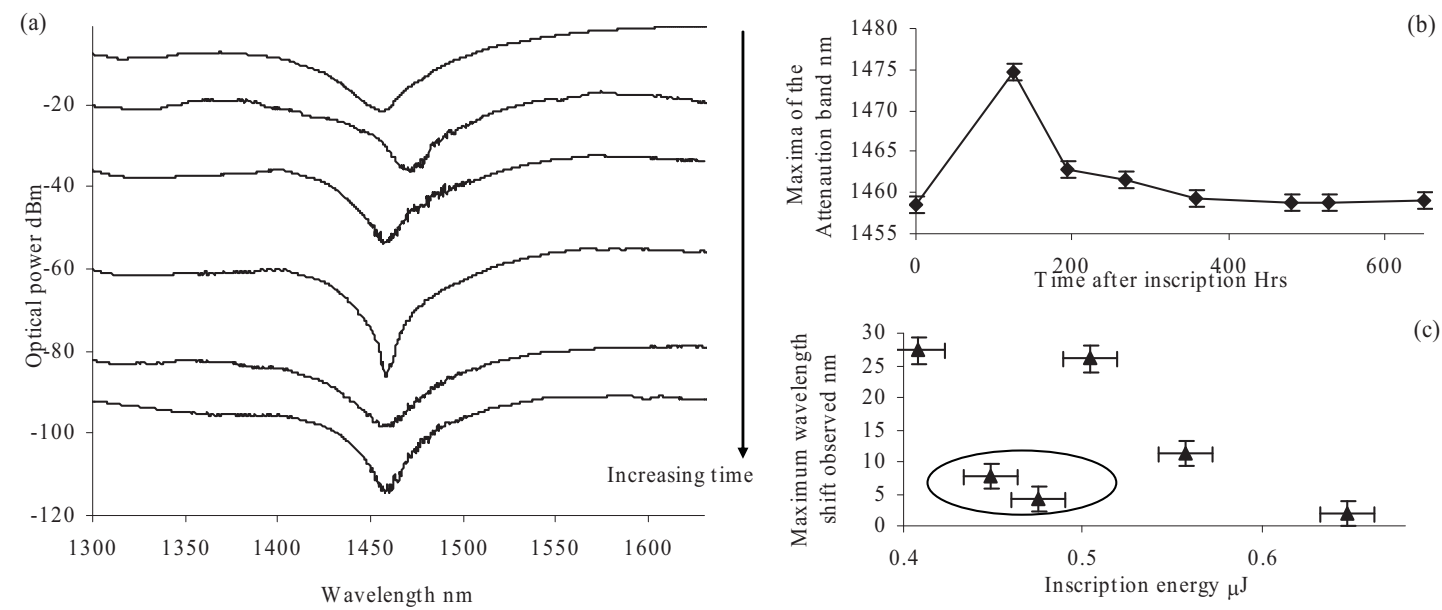

Figure 3. (a)Typical example of the post-fabrication spectral evolution at room temperature of the transmission spectrum of a femtosecond inscribed LPG written in ESM PCF. (b) The spectral response of the LPG as a function time. (c) The maximum red wavelength shift observed with the LPGs fabricated in the ESM PCF as a function of inscription power.

After approximately 600 hours of room temperature annealing, the LPGs appeared to be spectrally stable but there were red and blue wavelength shifts compared to the resonant wavelength immediately following fabrication, which appear to be related to the inscription energy, see figure 4a. Also there were variations observed in the polarisation induced wavelength shift of both bands, which appear to be related to both the energies used for inscription and how symmetric the inscription was, see figure $4 \mathrm{~b}$.
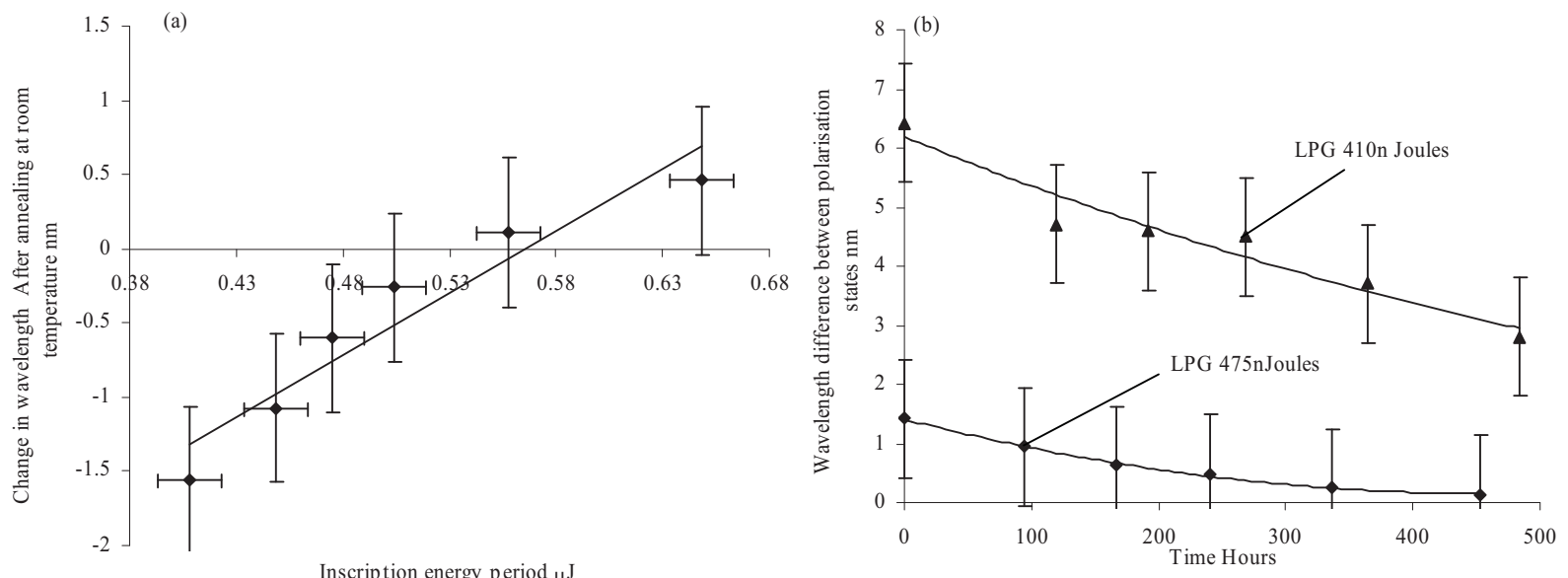

Figure 4. (a) The resultant wavelength shift after annealing at room temperature (b) The change in polarisation dependency with annealing at room temperature.

\section{Annealing studies at $80^{\circ} \mathrm{C}$}

Dramatic changes were observed for the LPG annealed at $80^{\circ} \mathrm{C}$, both in wavelength shift, strength and profile of the attenuation bands, see figures $5 \mathrm{a}$ and $5 \mathrm{~b}$. The largest resultant post-annealing wavelength shift of $23 \mathrm{~nm}$ was observed with the lowest inscription energies for the LPGs, see figure 6a. The strengths of the LPGs vary with this annealing condition with the highest reduction in coupling also seen with the lowest inscription energies (an $8 \mathrm{~dB}$ reduction, see figure $6 \mathrm{~b}$ ). Also it was noted that the time taken to obtain a steady-state spectrum varied from 60 hours for the LPG inscribed with $475 \mathrm{~nJ}$ to 150 hours for the LPG inscribed with $650 \mathrm{~nJ}$. 
(a)

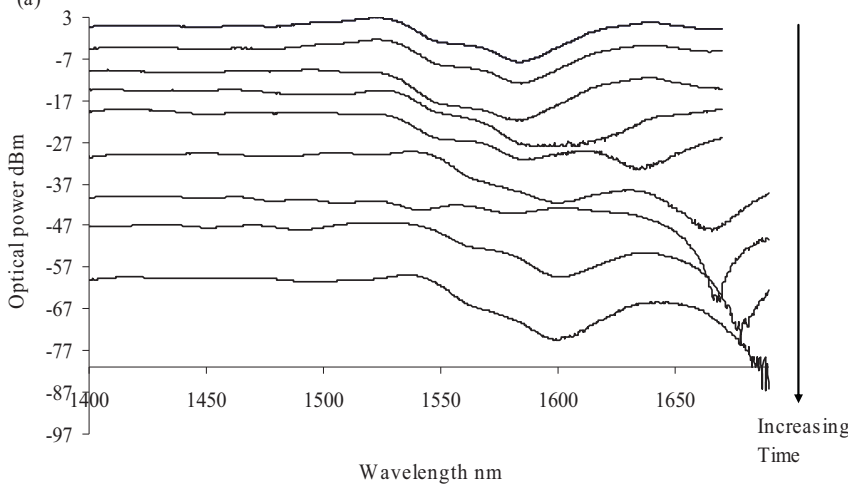

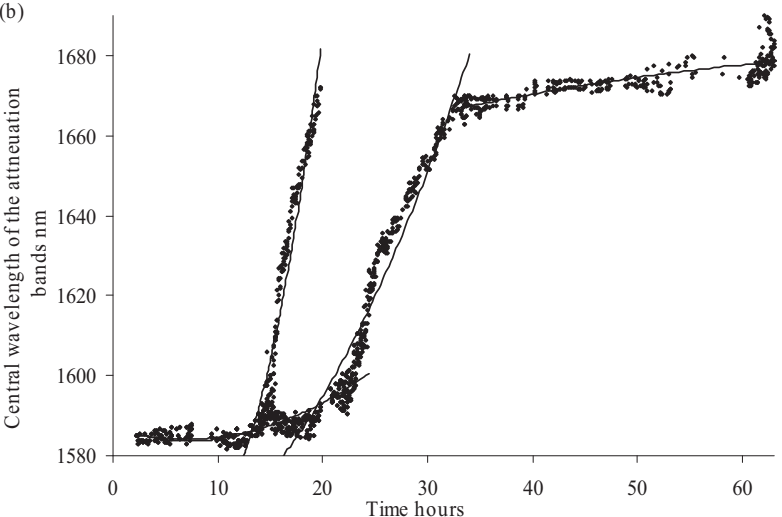

Figure 5. Annealing at $80^{\circ} \mathrm{C}$.(a)The temporal evolution of the transmission spectrum of a LPG written in ESM PCF (period $400 \mu \mathrm{m}$, inscription energy $475 \mathrm{~nJ}$ ). (b) The spectral evolution during annealing of the central wavelength of the attenuation band of the LPG (period $400 \mu \mathrm{m}$, inscription energy 470nJ).
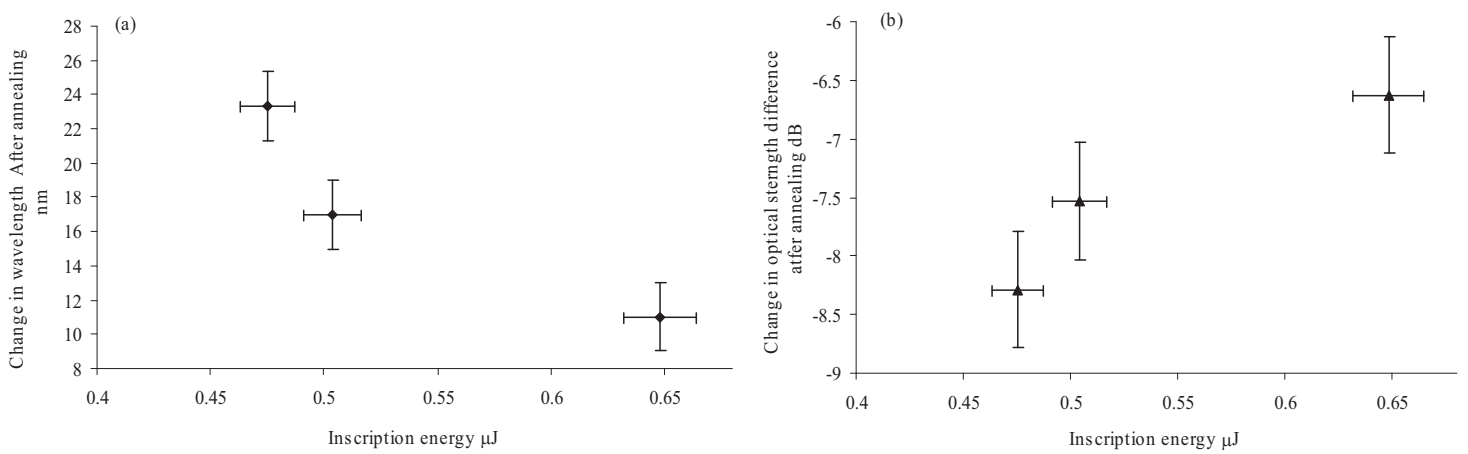

Figure 6. (a) The resultant wavelength shift after annealing at $80{ }^{\circ} \mathrm{C}$ as a function of inscription energy. (a) The resultant change in optical strength of the attenuation band after annealing $80^{\circ} \mathrm{C}$ as a function of inscription energy.

\section{DISCUSSION}

One of the main reasons for using femtosecond inscribed LPGs is their ability to operate at high temperatures ${ }^{10}$ but some recent studies $^{9}$ have suggested this may not be without problems. The current study has shown that the LPGs' thermal annealing properties are complex and intimately linked to their fabrication conditions, such as the inscription energy. This behaviour is manifested as postfabrication spectral evolution, both red and blue wavelength shifts at room temperature, depending upon inscription energy with the greatest shift observed, $1.5 \mathrm{~nm}$, corresponding with the lowest energy; $410 \mathrm{~nJ}$. The room temperature annealing results suggest that there is an optimum energy for minimal wavelength shift at room temperature; approximately $570 \mathrm{~nJ}$ for our arrangement. The results obtained so far for the higher annealing temperature $\left(80^{\circ} \mathrm{C}\right)$ have revealed red wavelength shifts, with the highest again occurring with the lowest inscription energy. The results so far suggest that greater understanding is required of the index modification mechanism of the LPGs to prevent misinterpretation of data from sensing applications.

\section{References}

1 V. Bhatia, A.M. Vengsarkar, "Optical fibre long-period grating sensors", Opt. Lett., vol.21, no.9, pp.692-694, 1996.

2 J. Petrovic et al, "Sensitivity of LPGs in PCFs Fabricated by an Electric Arc to Temperature, Strain, and External Refractive Index", J. Lightwave Technol, Vol.25, no.5, pp. 1306-1312

3 Y. Youk et al, "Refractive Index Profiling of a Core-Doped Photonic Crystal Fiber", PHOTONICS TECH. LETS, Vol. 19, No. 11, pp.87, 2007

4 T. Wu et al, “A Novel Ultraflattened Dispersion Photonic Crystal Fiber, “,”, PHOTONICS TECH. LETS, Vol. 17, No. 1, pp.67, 2005

5 V. Bhatia, "Applications of long-period gratings to single and multi-parameter sensing", Opt. Express., 1999, vol. 4, no. 11, pp.457-466

$6 \mathrm{H}$ Dobb, et al, "Gratings in Novel Fibre Geometries for Application in Shape Sensing", IoP In-fibre Gratings and Special Fibres, Stoneleigh,

Warwickshire (oct 2003)

7 K. Morishita, et al, "Fabrication and Resonance Wavelengths of Long-Period Gratings Written in a Pure-Silica Photonic Crystal Fiber by the Glass Structure Change", Lightwave Technol., Vol. 22, No. 2, pp.625, 2004

8 A. Martinez, et al, "Point by point FBG inscription by a focused NIR femtosecond laser", OSA Conf.Proc. CLEO/IQEC and PhAST Washington, DC, 2004, CMY6

9 T. Allsop, et al, "A comparison of the spectral properties of high temperature annealed long period gratings inscribed by fs laser, UV, and fusionarc", SPIE Vol 6193, Photonics Europe, Strasburg, France, 2006.

10 V. R. Bhardwaj, et al, "Stress in femtosecond-laser-written waveguides in fused silica", Opt. Letts, Vol. 29, No. 12, pp.1312, 2004 